\title{
Joint and Partial Carrier Components Assignment Techniques Based on User Profile in LTE Systems
}

\author{
Husnu S. Narman and Mohammed Atiquzzaman \\ School of Computer Science, University of Oklahoma, Norman, OK 73019 \\ Email: \{husnu, atiq\}@ou.edu
}

\begin{abstract}
The number of mobile users is rapidly increasing. Therefore, the bandwidth demand of mobile users significantly growing. To answer users' demand, Carrier Aggregation is proposed in LTE systems. In Carrier Aggregation, communication between users and base stations are achieved by multi bands which have different coverage areas and mobile users can simultaneously connect one or multi carrier components in each band. Because of mobility of users and quality of carriers, the best available Carrier Components of each band should be assigned to each user in order to provide desired service to users. Several works have been proposed in the literature to address Carrier Components assignment methods in LTE systems by using Channel Quality Indicator, quality of service and service types. Although the previous works on carrier assignment methods significantly increase the performance of LTE system, continuously increasing bandwidth demand of users forces the operators to manage resource allocation more intelligently. Therefore, we have proposed a novel Carrier Component assignment method which considers user profiles and channel quality indicator to increase quality of services and experiences getting by mobile users. Results show that the proposed method uses system resources efficiently and can improve performance of LTE systems. Our method will help service providers build efficient carrier components assignment methods through considering user profile and performance metrics, such as band usage, throughput and delay.
\end{abstract}

Index Terms-LTE, user profile, carrier component assignment, resource allocation, analysis.

\section{INTRODUCTION}

An increasing number of mobile users [1] request enormous amount of data over the Internet. Therefore, the bandwidth demand for mobile users is increasing exponentially [2]. To answer the bandwidth demand of mobile users, Carrier Aggregation $(\mathrm{CA})$ is proposed to extend bandwidth and support 1.5 Gbps for uplink and $3 \mathrm{Gbps}$ for downlink peak data rates in LTE systems [3]. In CA, communication between mobile users and base stations (eNodeB) are achieved by one or multiple Carrier Components (CC) in bands. One to two of $\mathrm{CCs}$ are primary carrier components (PCC), and can only be updated during handover [3], and the rest of CCs are considered as Secondary Carrier Components (SCCs).

Either all or some carriers for SCCs can be reassigned to each user during the carrier assignment process [4]. For example, while $i^{\text {th }}$ User Equipment $\left(\mathrm{UE}_{\mathrm{i}}\right)$ is leaving from Band-c communication range to enter Band- $b$ communication range, it is possible to simultaneously reassigning all or some carrier components of SCCs to $\mathrm{UE}_{\mathrm{i}}$. Simultaneously reassigning all carriers causes delay for packets of $\mathrm{UE}_{\mathrm{i}}$ which are waiting for service. However, it may increase the overall performance of LTE systems if CQI Indexes of new SCCs are higher than the previous SCCs (Reassigning all carriers to a User Equipment (UE) at the same time is called as Joint Carrier Components Assignment (JCCA).) On the other hand, only updating carriers of Band-c by allowing carrier components of Band- $b$ or Band- $a$ to continue serving $\mathrm{UE}_{\mathrm{i}}$, can be another way to prevent packets experiencing delay or drop during the assignment process. However, finding better carriers for SCCs in current position may be possible for this user to have better service (Reassigning some of carriers to a UE is called as Partial Carrier Components Assignment (PCCA)) [4]. Further reading about joint and partial carrier assignment techniques can be found in [4].

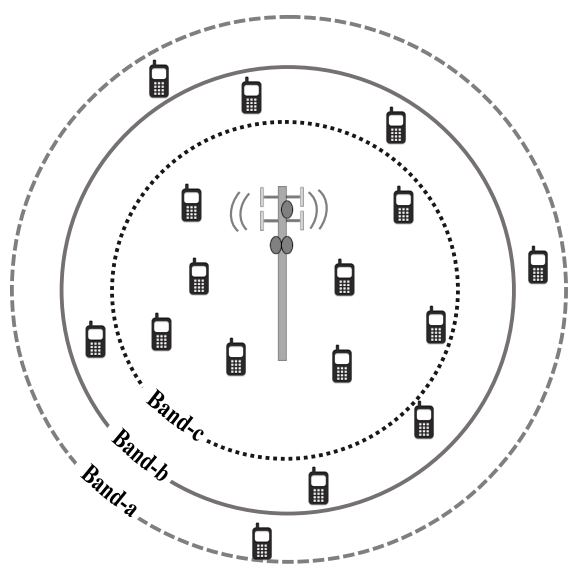

Fig. 1: eNodeB (eNB) with multi bands and several UEs.

Fig. 1 shows the multi-band system in mobile networks. In this system, each band may have different communication range (i.e., here range means coverage). A UE can simultaneously connect one or multi bands as illustrated in Fig. 1. To use system resources efficiently and provide better service to users, base stations must assign one or multi CCs to UEs by considering specifications of each UE, the number of UEs in the system, Channel Quality Indicator (CQI) for carrier components, etc. If carrier assignment method is not well developed, the performance of system significantly decreases [5].

Because of recent improvements in LTE systems, there are several proposed carrier assignment methods [6]-[19] in the literature. In [12], a method is proposed to measure the Channel Quality Indicator (CQI) in LTE. In [6]-[9], full or partial 
feedback is used for CQI to find the best available carriers for each user. In [11], [19], distribution of carriers to users are balanced. In [10], [14], [17], uplink CA has been proposed by considering a ratio function, traffic type and CQI to increase throughput while sending data from users to eNodeB (eNB). While uplink carrier assignment has bandwidth and power limitation, downlink carrier assignment has only bandwidth limitation. In [13], [16], service-based methods are proposed by giving priority for some services while assigning carriers to users. In [15], multi carriers are assigned to users in such a way that delay time is decreased. In addition to the above methods, there exist traditional carrier assignment methods, Least Load (LL) (LL is also called as Round Robin (RR)) and Random (R) [18]. LL well balances traffic loads across different carriers in short and long terms while $\mathrm{R}$ only well balances traffic loads across different carriers in long term. However, the both ignore CQI and Quality of Service (QoS) requirements of each user.

Continuously increasing bandwidth demand of users forces the operators to manage traffic more intelligently because economic and physical limitations do not allow operators to extend network capacity [20]. Although load balancing, QoS and CQI methods, as summarized above, have been used to manage carrier assignment process, more advance techniques [20] in addition to these methods will be needed to satisfy users' demands in LTE systems. Therefore, in [21], we have developed user profile carrier assignment method in addition to traffic types to manage carrier assignment more intelligently in LTE systems because not only mobility of each user profile is different but also each user profile needs different QoS from different types of traffic [20]. As illustrated

TABLE I: Mobile Users Profile

\begin{tabular}{|c|c|c|c|c|c|c|c|}
\hline & & & \multicolumn{5}{|c|}{ User Profile } \\
\hline & & & Teen. & H. wife & B. man & Grad. Stu. & G. parent \\
\hline \multirow{9}{*}{ 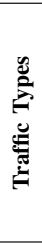 } & \multirow{4}{*}{$\simeq$} & Video & V. High & Middle & Low & Medium & Low \\
\hline & & Onl. Game & V. High & Low & Low & Medium & Low \\
\hline & & Movie & V. High & V. High & Low & Medium & Low \\
\hline & & Talk & Low & Medium & High & Medium & V. High \\
\hline & \multirow{3}{*}{$\bar{z}$} & Web & High & Low & V. High & Medium & Low \\
\hline & & Mail & High & Low & V. High & Medium & Low \\
\hline & & SMS & V. High & Medium & Low & Medium & Low \\
\hline & \multirow{2}{*}{$\dot{\Xi}$} & Mobility & Low & Medium & V. High & Low & Low \\
\hline & & Location & Low & Medium & High & Medium & Low \\
\hline
\end{tabular}

in Table I, bandwidth requirements of each application (Real Time (RT) and Non-real Time (NRT) services) and mobility are different for each user profile (See Table I for Teenager and Businessman). Therefore, user profile, in addition to CQI, can be considered to increase QoS and Quality of Experience (QoE).

None of the above works except our primary reports [21], [22] considers user profile for carrier assignment. In [21], [22], we have shown the benefits of the user profile carrier assignment method by integrating user profile in $\mathrm{R}$ method. However, neither we have considered CQI and load balance properties for user profile carrier assignment method nor joint and partial carriers assignment techniques [4], [22] are investigated in user profile carrier assignment method. However, it is essential to consider CQI and load balance properties with the aforementioned techniques to improve and evaluate user profile carrier assignment method. Therefore, the aim of this work is to improve user profile carrier assignment method by considering CQI and load balance properties, and evaluate the performance of user profile carrier assignment methods by using joint and partial carriers assignment techniques.

The objective of this paper is to increase the performance of carrier assignment process by considering user profiles of mobile users in LTE systems. The key contributions of this work are as follows: (i) defining user profiles with respect to traffic types and mobility, (ii) proposing a carrier assignment algorithm based on user profiles and CQI by using LL method, and (iii) evaluating performance of the proposed method by using joint and partial carrier assignment techniques with extensive simulation.

Results show that the proposed method uses system resources efficiently and can improve performance of LTE systems. Therefore, the proposed method and related analysis will help service providers build carrier components assignment methods through considering user profile and performance metrics, such as band usage, throughput and delay.

The rest of the paper is organized as follows. In Section II, we explain the system model and user profile with its properties. The proposed method is presented in Section III and simulation environments with parameters are explained in Section IV. In Section V, simulation results are analyzed. Finally, Section VI has the concluding remarks.

\section{USER PROfile AND SyStem Model}

In Fig. 1, users are mobile and can connect one band or multi bands simultaneously based on coverages of bands and positions of users. Users can change connected bands to another band in same eNB if it moves from coverage of one band to coverage of another band. For example, when a user, which is using Band-b, enters Band-c communication range, some of several band selection scenarios with carrier assignment for the user can be as follows (see Fig. 1): (i) the user may need to use larger bandwidth for services, therefore changing its band to Band-c will increase performance, (ii) mobility of the user is high, therefore changing its band to Band-c may decrease performance because of low range, (iii) the user does not need to use larger bandwidth from Band-c, thus no need to update its band, and (iv) the mobility of the user is high and the user needs larger bandwidth, therefore it can use both bands.

In addition to band selection with carrier assignment, determining the number of required carrier components for each user is important because of power usage and QoS efficiency. For example, when a user can enter an eNB range, some of scenarios to determine the number of carriers for the user can be as follows: (i) data usage of the user is small, therefore only one carrier (one $\mathrm{CC}$ ) will be enough, (ii) the data usage of the user is high, therefore, assigning multi carriers will increase performance, and (iii) device type of the user is not allowed to assign more than one carrier, therefore, one carrier will 
be assigned. Above scenarios show the importance of carrier assignment in LTE systems in order to increase performance.

\section{A. User Profile Detection Based on Services}

Historical information of each UE plays a crucial role to identify user profiles. As shown in Table II, each UE holds Times, Connection Time (Con. T) and Idle Time (Idle T.), RT and NRT services data sizes for each eNB. In Table II, Times illustrates how often a UE connects to eNBs, Con. T represents how long a UE keeps connected eNBs and Idle $T$. gives how long UE connected but not receive any services from previous sessions for each band.

TABLE II: User Profile Detection Based on eNodeBs

\begin{tabular}{|c|c|c|c|c|c|c|c|}
\hline & \multicolumn{3}{|c|}{ Band-a/Band-b/Band-c } & \multicolumn{2}{c|}{ RT-Services } & \multicolumn{2}{c|}{ NRT-Services } \\
\hline eNB-ID & Times & Con. T. & Idle T. & Video & Game & Web & Mail \\
\hline$I D_{1}$ & $f_{1}$ & $c_{1}$ & $t_{1}$ & $v_{1}$ & $g_{1}$ & $w_{1}$ & $m_{1}$ \\
\hline$I D_{2}$ & $f_{2}$ & $c_{2}$ & $t_{2}$ & $v_{2}$ & $g_{2}$ & $w_{2}$ & $m_{2}$ \\
\hline$I D_{3}$ & $f_{3}$ & $c_{3}$ & $t_{3}$ & $v_{3}$ & $g_{3}$ & $w_{3}$ & $m_{3}$ \\
\hline$I D_{4}$ & $f_{4}$ & $c_{4}$ & $t_{4}$ & $v_{4}$ & $g_{4}$ & $w_{4}$ & $m_{4}$ \\
\hline$I D_{5}$ & $f_{5}$ & $c_{5}$ & $t_{5}$ & $v_{5}$ & $g_{5}$ & $w_{5}$ & $m_{5}$ \\
\hline$I D_{6}$ & $f_{6}$ & $c_{6}$ & $t_{6}$ & $v_{6}$ & $g_{6}$ & $w_{6}$ & $m_{6}$ \\
\hline$I D_{7}$ & $f_{7}$ & $c_{7}$ & $t_{7}$ & $v_{7}$ & $g_{7}$ & $w_{7}$ & $m_{7}$ \\
\hline$I D_{8}$ & $f_{8}$ & $c_{8}$ & $t_{8}$ & $v_{8}$ & $g_{8}$ & $w_{8}$ & $m_{8}$ \\
\hline
\end{tabular}

In order to identify user profile from Table II, some statistical analysis such as percentage, average, etc measurement, can be applied. For example, percentage of Connection Time of $\mathrm{UE}_{\mathrm{i}}$ to $\mathrm{eNB}_{\mathrm{j}}\left(\Delta C_{j}^{i}\right)$ and percentage of Times of $\mathrm{UE}_{\mathrm{i}}$ to $\mathrm{eNB}_{\mathrm{j}}$ $\left(\Delta T_{j}^{i}\right.$ ) can simply be calculated as follows:

$$
\Delta C_{j}^{i}=100 \times \frac{c_{j}}{\sum_{s=1}^{k} c_{s}} \text { and } \Delta T_{j}^{i}=100 \times \frac{f_{j}}{\sum_{s=1}^{k} f_{s}}
$$

where $k$ is the number of eNBs. Lower $\Delta T_{j}^{i}$ and higher $\Delta C_{j}^{i}$ indicate that $\mathrm{UE}_{\mathrm{i}}$ spends its more time around $\mathrm{eNB}_{\mathrm{j}}$ with specified carrier band. On the other hand, higher $\Delta T_{j}^{i}$ and lower $\Delta C_{j}^{i}$ indicate that $\mathrm{UE}_{\mathrm{i}}$ temporarily requests service from $\mathrm{eNB}_{\mathrm{j}}$. For example, $\mathrm{UE}_{\mathrm{i}}$ just uses $\mathrm{eNB}_{\mathrm{j}}$ while driving home, to work or school. More statistical analysis about user profile based on Table II can be found in [21], [22].

\section{B. System Model}

Fig. 2 shows a system model for a carrier assignment method and a queue scheduler. There are $n$ number of UEs and each UE can only connect up to $m$ number of carriers. One to two of carriers are PCC, and can only be updated during handover [3], but the rest of carriers are SCCs and can be updated according to JCCA and PCCA.

Today, UEs of LTE advanced system can simultaneously connect up to five carrier components [3]. However, assigning all five carriers to a UE can increase power consumption and interference. Therefore, it is important to have a carrier assignment method, which firstly determines the number of required carriers and band of each carrier for each UE. Determining the number of required carriers and band of each carrier for each UE do not only decrease power consumption and interference but also increase efficiency of resources usage. However, the

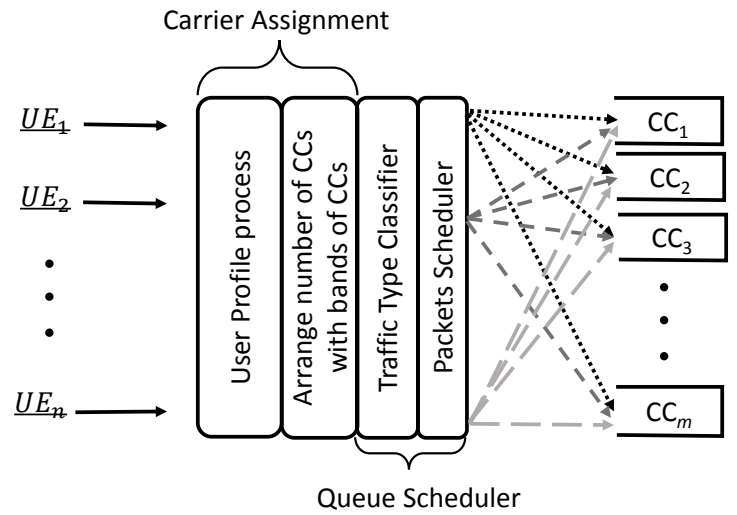

Fig. 2: System Model with $n$ users and $m$ available CCs.

only way is to do it by estimating data usage and mobility of UEs (user profiles). Estimating RT and NRT data usage for a UE helps an eNB arrange the number of carriers and their bandwidth sizes, and estimating mobility of a UE reduces handover overheads and risk of connection lost. However, a carrier assignment method based on only mobility and data usage of users can lead unbalance users loads on carriers. Therefore, LL carrier assignment method is integrated with user profile to balance users loads across carriers, and in order to eliminate CQI blind carrier assignment of LL, CQI awareness is added to the proposed assignment method in order to have an advance carrier assignment method.

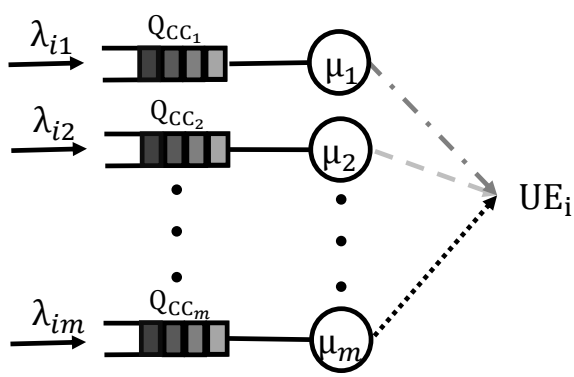

Fig. 3: Downlink System Model with one user and $m$ available CCs.

After carrier assignment process finishes, and the number of $\mathrm{CCs}$ and bands of each $\mathrm{CC}$ are determined, queue scheduler starts to transfer packet over CCs for a UE. We have used Disjoint Queue Scheduler [23] because of realistic approach for LTE systems. Disjoint Queue Scheduler allows all users to have disjoint buffers for each carrier as showed in Fig. 3.

\section{User Profile Carrier Components Assignment METHOD}

The proposed method considers three crucial parameters that enable dynamic CCs assignment: (i) UEs device types in terms of LTE and LTE-A. (LTE device can only connect one CC while LTE-A device can connect up to five CCs, (ii) CQI of CCs [6]-[9], and (iii) user profiles of UEs. 
A. Measurement of the Number of Required CCs with Their Bands for Each UE

In order to estimate the number of required CCs with their bands for $\mathrm{UE}_{\mathrm{i}}$ in $\mathrm{eNB}_{\mathrm{j}}$, total data usage and connection time which are obtained from Table II and number of active users in each band are used.

Firstly, selection of bands is made based on following rate function which depends on mobility of $\mathrm{UE}_{\mathrm{i}}$ and busyness of bands;

$$
{ }^{x} \alpha_{\text {rate }}=\frac{{ }^{x} \eta_{u}}{{ }^{x} \beta_{u}} *{ }^{x} c_{i j}
$$

where $x \in a, b, c$ bands, ${ }^{x} \eta_{u}$ is maximum capacity of Band-x in terms of users, ${ }^{x} \beta_{u}$ is active users on Band-x, and ${ }^{x} c_{i j}$ is connection time of $\mathrm{UE}_{\mathrm{i}}$ to $\mathrm{eNB}_{\mathrm{j}}$ by using Band-x. After ${ }^{x} \alpha_{\text {rate }}$ rate is measured for each band, the band which has maximum rate is selected for $\mathrm{UE}_{\mathrm{i}}$. If rates of two or three bands are same, two of them or three of them are selected, respectively. Secondly, the number of required CCs is measured by considering active users in selected bands and total data usage of $\mathrm{UE}_{\mathrm{i}}$ on $\mathrm{eNB}_{\mathrm{j}}$ as follow:

$$
{ }^{n u m} C C=\frac{{ }^{x} \varphi_{i j}}{{ }^{x} \eta_{p}}
$$

where $x \in a, b, c$ bands, ${ }^{x} \eta_{p}$ is maximum capacity of Band-x in terms of packets, ${ }^{x} \beta_{p}$ is measured by summing all packet arrival rates of active users on Band-x, and ${ }^{x} \varphi_{i j}$ is average data usage of $\mathrm{UE}_{\mathrm{i}}$ in $\mathrm{eNB}_{\mathrm{j}}$ by using Band-x. Note that ${ }^{x} \eta_{p}$ depends on CQI of each active user and bandwidth size. If ${ }^{\text {num }} C C$ is greater than five or service capacity limitation (e.g. limitation of payment), ${ }^{\text {num }} C C$ is restricted according to the limitations. By using Eqs. (2) and (3), bands and the required number of CCs are determined.

\section{B. Carrier Assignment Process}

By using ${ }^{x} \alpha_{\text {rate }}$ and ${ }^{n u m} C C$, the proposed carrier assignment method is processed as follows for $\mathrm{UE}_{\mathrm{i}}$ in $\mathrm{eNB}_{\mathrm{j}}$ : (i) $\mathrm{UE}_{\mathrm{i}}$ device capacity information is transferred to $\mathrm{eNB}_{\mathrm{j}}$. (ii) Partially or fully CQI feedback is obtained. Then bands for carriers are selected and the required number of CCs from the selected bands are calculated by using Eqs. (2) and (3), respectively. (iii) The number of carriers is equally divided if multiple bands are selected (i.e., if the required number of $C C s$ is four and the selected bands are Band- $a$ and Band-b, two $\mathrm{CCs}$ are assigned from each band to $\mathrm{UE}_{\mathrm{i}}$ ) (iv) Firstly, carriers which have the least number of active users (Least Load) and carriers of which CQI Indexes are higher than a threshold are selected from bands until ${ }^{\text {num }} C C$ is reached. If ${ }^{n u m} C C$ is not reached, additionally carriers which have the less number of users from other carriers are selected until the number of selected CCs is reached to ${ }^{n u m} C C$. (v) Assignment of the selected CCs is finshed and buffers for each carrier are created for $\mathrm{UE}_{\mathrm{i}}$. (vi) Repeating process until all users are allocated.

\section{Simulation of the System}

Discrete event simulation for the downlink carrier assignment process has been implemented in Matlab by considering the carrier assignment method which are mentioned in Sections II and III.

\section{A. Assumptions for eNBs}

It is assumed that there is only one eNB which has three bands to provide service to users. Some parameters of eNB are summarized in Table III.

\section{TABLE III: Table of eNB Parameters}

\begin{tabular}{lll}
\hline Num. of eNB & $\triangleq$ & 1 \\
Used Bands & $\triangleq$ & $800 \mathrm{MHz}, 1.8 \mathrm{GHz}, 2.6 \mathrm{GHz}$ \\
Num. of CCs in each band & $\triangleq$ & 4 \\
Total Num. of CCs & $\triangleq$ & 12 \\
Queue Length of all $\mathrm{Q}_{\mathrm{CC}}$ & $\triangleq$ & $\triangleq$ \\
Bandwidth size of CCs & $\triangleq$ & packets \\
Modulations & $\triangleq$ & $\mathrm{MHz}$ \\
CQI Index & $\triangleq$ & $\triangleq$ \\
Transmission Time Interval, 7 , and 11 \\
Time for CCA & $\triangleq 1 \mathrm{~ms}$ \\
CQI Index threshold & $\triangleq$ & $\triangleq 5$ \\
\hline
\end{tabular}

\section{B. Assumptions for UEs}

There are LTE and LTE-A types equipment in the system. The number of both type equipment is equal. However, LTE type equipment can only use one CC and LTE-A type equipment can use multiple CCs. In simulation, four CCs can be simultaneously used by LTE-A type equipment because maximum five CCs can be used by LTE-A type equipment, and one of them must be used for primary carrier component (see Section II). Initially users are uniformly distributed in area. $50 \%$ of users can randomly move around of the eNB in specified time interval. Each user can only download one type of traffic. Packet arrivals follow Poisson distribution and packet arrival rates of traffic are enlarged when the number of users is increased. Selected Transmission Time Interval (TTI) for a packet is $1 \mathrm{~ms}$.

\section{Packet Scheduling}

The result cannot be obtained without packet scheduler. Therefore, minimum delay packet scheduling is used in the simulation. Packet arrival rate of each UE are kept same for all test cases. CQI Index for all carriers can be one of four options which are given in Table III because of UEs and the eNB positions. Each packet is transferred by using one of assigned carriers which minimizes packet delay. If there is no available assigned carriers to serve arrived packets, packets are enqueued to corresponding carriers queues (assigned CCs queues for each UE) based on minimum delay measurement. If there is no spaces in queues, arrived packets are dropped. 


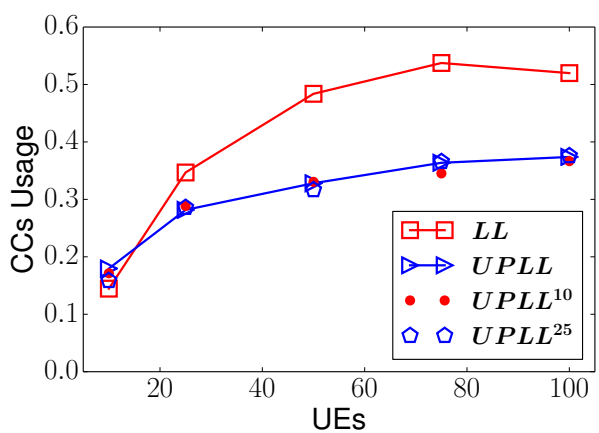

Fig. 4: CCs usage of LL and UPLLs by using joint assignment technique.

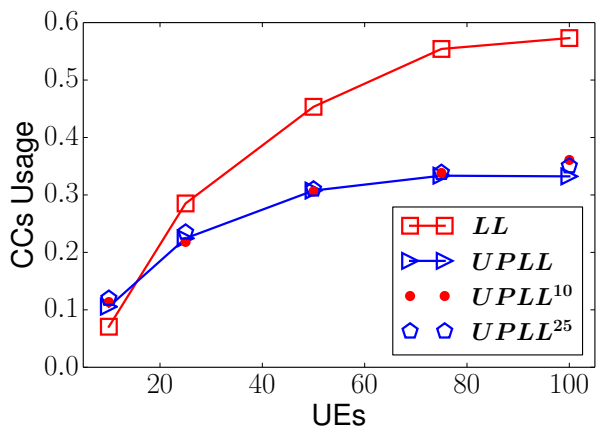

Fig. 7: CCs usage of LL and UPLLs by using partial assignment technique.

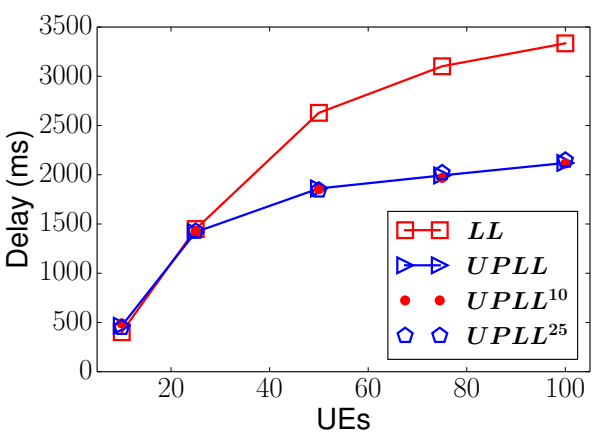

Fig. 5: Delay of LL and UPLLs by using joint assignment technique.

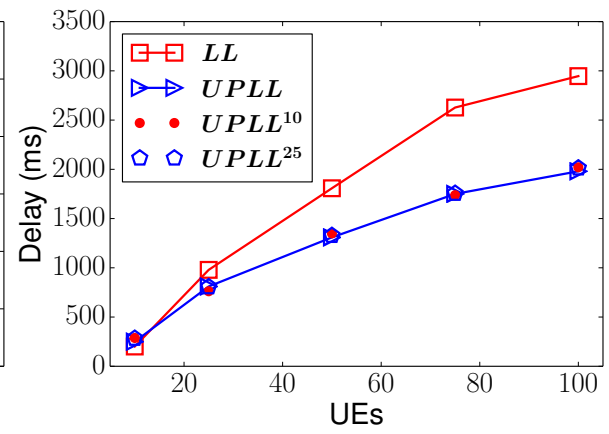

Fig. 8: Delay of LL and UPLLs by using partial assignment technique.

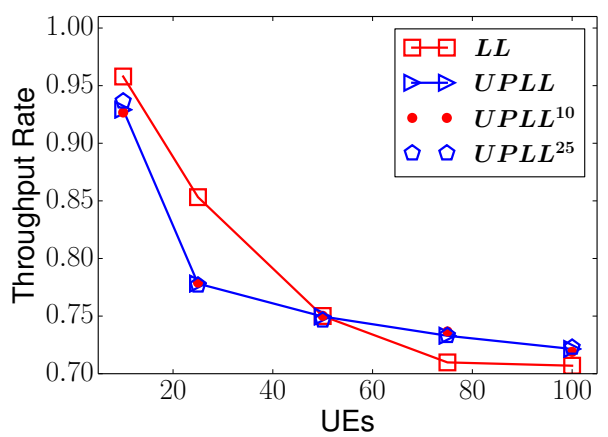

Fig. 6: Throughput of LL and UPLLs by using joint assignment technique.

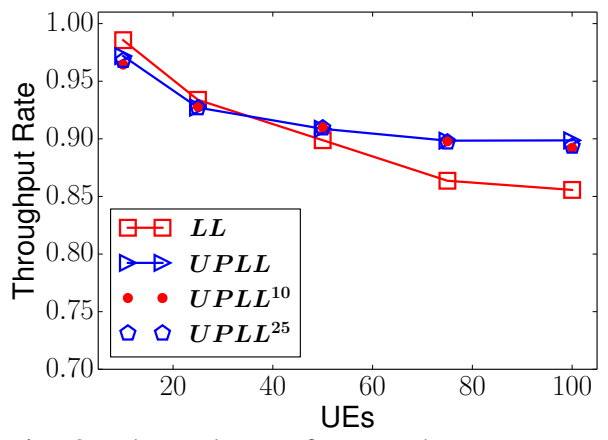

Fig. 9: Throughput of LL and UPLLs by using partial assignment technique.

\section{Observation Methodology}

The results in Section $\mathrm{V}$ are average of 200 realizations for different number of users. The impact of light and heavy users loads on User profile and modified LL is investigated by using joint and partial carrier assignment techniques. As explained above, LL method is selected because LL well balances distribution of users to carriers. Modified LL uses LL and additionally CQI for carrier assignment (see (iv) in III-B). To have fair comparison, modified LL is compared with the proposed user profile carrier assignment method instead of LL. Modified Least Load (LL), and User Profile Least Load (UPLL), User Profile Least Load based on $10 \%$ error of user profile estimation (UPLL ${ }^{10}$ ) and User Profile Least Load based on $25 \%$ error of user profile estimation (UPLL ${ }^{25}$ ) carrier assignment methods have been analyzed for joint and partial carrier assignment techniques. Error means that data usage is estimated based on these above error percentages. For example, a UE data usage rate is $100 \mathrm{MB}$ but estimated data usage of the UE can be $125 \mathrm{MB}$ or $75 \mathrm{MB}$ for UPLL ${ }^{25}$ and 110 or 90 for UPLL ${ }^{10}$. Therefore, the proposed method is evaluated under more realistic scenario.

We present the performance of LL and UPLLs (i.e., here UPLLs means UPLL, UPLL ${ }^{10}$, and $\mathrm{UPLL}^{25}$ ) by comparing system CCs usage rate, throughput rate and delay. CCs usage rate of each band is measured by dividing total packets of active users on each carrier to total capacity of carriers for each discrete time then the system CCs usage rate is obtained by averaging the measured CCs usage rate of each band. Throughput rates are measured by dividing transferred packets to all generated packets. Therefore, while the number of users is increased, throughput rate per user decreases because of carriers capacities. Block rate is not given because it is just inverse of throughput rate. Delay for each packet is determined based on waiting time in queues and servers. Then overall delay of the system is obtained by summing delay of each packet. As a result of these evaluations, trade off between resource usage and managed QoS are compared.

\section{RESUlts}

In this section, overall system performances are given for LL and UPLLs by using joint and partial carrier assignment techniques.

\section{A. CCs Usage}

Figs. 4 and 7 show CCs usage rate of LL and UPLLs for joint and partial carrier assignment techniques, respectively. CCs usage rate gradually increases when the number of users is getting larger for all cases. Though large number of users, CCs usage rate of all cases do not reach peak rate $(=1)$ because uniform distribution of users around eNB decreases the number of users in Band-b and Band-c communication ranges. Thus, $\mathrm{CCs}$ usage rate does not reach peak rate although CCs usage rate in Band- $a$ is high. Moreover, CCs usage rate of UPLLs is lower than CCs usage rate of LL for both joint and partial assignment techniques and CCs usage rate of LL and UPLLS in joint carrier assignment technique are almost equal to CCs usage rate of LL and UPLLs in partial carrier assignment technique, respectively. Interestingly, CCs usage 
rate of UPLLs are almost equal for both joint and partial carrier assignment techniques.

\section{B. Delay}

Figs. 5 and 8 demonstrate delay of LL and UPLLs for joint and partial carrier assignment techniques, respectively. While the number of users is increased, delay is regularly getting higher for all cases. However, delay of joint carrier assignment technique is greater than delay of partial carrier assignment technique in LL and UPLLs methods and delay of LL is higher than delay of UPLLS in both joint and partial carrier assignment techniques.

\section{Throughput}

Figs. 6 and 9 depict throughput rate of LL and UPLLs for joint and partial carrier assignment techniques, respectively. Increment in the number of users gradually reduces throughput rate for all cases. However, throughput rate of partial carrier assignment technique is greater than throughput of joint carrier assignment technique for LL and UPLLs. Moreover, throughput rate is higher in UPLLs than in LL when the number of users is greater than 50 and is a little lower in UPLLs than in LL otherwise for joint and partial carrier assignment techniques.

\section{Summary of Results}

Based on the results, we make the following observations: (i) partial carrier assignment technique is better than joint carrier partial assignment technique for modified LL and UPLLs, (ii) UPLLs is better than modified LL in terms of CCs usage rate and delay, and UPLLs increase throughput rate when the number of users is high, and (iii) increasing error percentage of user profile does not affect over all performance of UPLLs (see Figs. 4-9 for UPLL, UPLL ${ }^{10}$, and UPLL ${ }^{25}$ ).

\section{CONCLUSION}

In this paper, we have proposed a carrier component assignment method for LTE systems by considering user profiles and CQI. Band usage, delay and throughput rate of systems have been demonstrated for the proposed method by using joint and partial carrier assignment techniques through extensive simulations. Results show that the proposed method uses system resources efficiently and improve performance of LTE systems. Our proposed method and related analysis will help service providers build efficient carrier assignment methods by considering user profile and performance metrics, such as band usage, throughput and delay.

\section{REFERENCES}

[1] F. Richter. (2013, Sep) Smartphone sales break the billion barrier. Accessed: June 12, 2014. [Online]. Available: http://www.statista.com/ chart/777/global-connected-device-shipments/

[2] H. Singh, J. Hsu, L. Verma, S. S. Lee, and C. Ngo, "Green operation of multi-band wireless LAN in $60 \mathrm{GHz}$ and $2.4 / 5 \mathrm{GHz}$," in Consumer Communications and Networking Conference (CCNC), Las Vegas, NV, Jan 9-12, 2011, pp. 787-792.

[3] J. Wannstrom. (2013, June) LTE-Advanced. [Online]. Available: http://www.3gpp.org/technologies/keywords-acronyms/97-lte-advanced
[4] H. S. Narman and M. Atiquzzaman, "Analysis of Static Partial Carrier Components Assignment in LTE Systems," University of Oklahoma, Tech. Rep., Sep 2014. [Online]. Available: http://www.cs. ou.edu/ netlab/TR/Lte-Cc-Static-TR-OU-TNRL-14-101.pdf

[5] I. F. Akyildiz, D. M. Gutierrez-Estevez, and E. C. Reyes, "The evolution to 4G cellular systems: LTE-Advanced," Physical Communication, vol. 3, pp. 217-244, Mar 2010.

[6] L. xiang Lin, Y. an Liu, F. Liu, G. Xie, K. ming Liu, and X. yang Ge, "Resource scheduling in downlink LTE-Advanced system with carrier aggregation," The Journal of China Universities of Posts and Telecommunications, vol. 19, no. 1, pp. 44 - 49, Feb 2012.

[7] N. Kolehmainen, J. Puttonen, P. Kela, T. Ristaniemi, T. Henttonen, and M. Moisio, "Channel quality indication reporting schemes for UTRAN long term evolution downlink," in IEEE Vehicular Technology Conference, Singapore, May 11-14, 2008, pp. 2522-2526.

[8] S.-B. Lee, S. Choudhury, A. Khoshnevis, S. Xu, and S. Lu, "Downlink MIMO with frequency-domain packet scheduling for 3GPP LTE," in INFOCOM, Rio de Janeiro, Apr 19-25, 2009, pp. 1269-1277.

[9] S. Donthi and N. Mehta, "Performance analysis of subband-level channel quality indicator feedback scheme of LTE," in National Conference on Communications, Chennai, Jan 29-31, 2010.

[10] H. Yang, F. Ren, C. Lin, and J. Zhang, "Frequency-domain packet scheduling for 3GPP LTE uplink," in INFOCOM, San Diego, CA, Mar 14-19, 2010.

[11] Y. Wang, K. Pedersen, T. Sorensen, and P. Mogensen, "Carrier load balancing and packet scheduling for multi-carrier systems," IEEE Transactions on Wireless Communications, vol. 9, no. 5, pp. 1780-1789, May 2010.

[12] X. Chen, H. Yi, H. Luo, H. Yu, and H. Wang, "A novel CQI calculation scheme in LTE $\backslash$ LTE-A systems," in International Conference on Wireless Communications and Signal Processing, Nanjing, Nov 9-11, 2011.

[13] F. Liu, W. Xiang, Y. Zhang, K. Zheng, and H. Zhao, "A novel QoEbased carrier scheduling scheme in LTE-Advanced networks with multiservice," in Vehicular Technology Conference, Quebec City, Canada, Sep 3-6, 2012.

[14] H. K. Rath, M. Sengupta, and A. Simha, "Novel transport layer aware uplink scheduling scheme for LTE-based networks," in National Conference on Communications, New Delhi, India, Feb 15-17, 2013.

[15] S. Bodas, S. Shakkottai, L. Ying, and R. Srikant, "Scheduling for small delay in multi-rate multi-channel wireless networks," in INFOCOM, Shanghai, China, Apr 10-15, 2011.

[16] W. Fu, Q. Kong, W. Tian, C. Wang, and L. Ma, "A QoS-aware scheduling algorithm based on service type for LTE downlink," in International Conference on Computer Science and Electronics Engineering, Hangzhou, China, Mar 22-23, 2013, pp. 2468-2474.

[17] R. Sivaraj, A. Pande, K. Zeng, K. Govindan, and P. Mohapatra, "Edgeprioritized channel- and traffic-aware uplink carrier aggregation in LTEAdvanced systems," in International Symposium on a World of Wireless, Mobile and Multimedia Networks, San Francisco, CA, June 25-28, 2012.

[18] T. Dean and P. Fleming, "Trunking efficiency in multi-carrier CDMA systems," in 56th Vehicular Technology Conference, Vancouver, Canada, Sep 24-28, 2002, pp. 156-160.

[19] T. Girici, C. Zhu, J. R. Agre, and A. Ephremides, "Proportional fair scheduling algorithm in OFDMA-based wireless systems with QoS constraints," Journal of Communications and Networks, vol. 12, pp. 3042, 2010.

[20] Ixia. (2013, Dec) Quality of service (QoS) and policy management in mobile data networks. White Paper, Accessed: July 10, 2014. [Online]. Available: http://www.ixiacom.com/pdfs/library/white_papers/ policy_management.pdf

[21] H. S. Narman and M. Atiquzzaman, "User Profile Carrier Components Assignment Method in LTE Systems," University of Oklahoma, Tech. Rep., Oct 2014. [Online]. Available: http://www.cs.ou.edu/ netlab/TR/ User-Profile-TR-OU-TNRL-14-102.pdf

[22] H. Narman and M. Atiquzzaman, "Carrier components assignment method for LTE and LTE-A systems based on user profile and application," in IEEE International Broadband Wireless Access (BWA) GLOBECOM Workshop, Austin, TX, Dec. 12, 2014.

[23] L. Chen, W. Chen, X. Zhang, and D. Yang, "Analysis and simulation for spectrum aggregation in lte-advanced system," in 70th Vehicular Technology Conference Fall, Anchorage, AK, Sep 20-23, 2009. 